\title{
Tombes et cimetières éthiopiens : des rois, des
} saints, des anonymes

\section{Marie-Laure Derat}

\section{(2) OpenEdition}

1 Journals

\section{Édition électronique}

URL : http://journals.openedition.org/abpo/459

DOI : $10.4000 / a b p o .459$

ISBN : 978-2-7535-1508-6

ISSN : 2108-6443

\section{Éditeur}

Presses universitaires de Rennes

Édition imprimée

Date de publication : 30 décembre 2007

Pagination : 43-55

ISBN : 978-2-7535-0598-8

ISSN : 0399-0826

\section{Référence électronique}

Marie-Laure Derat, «Tombes et cimetières éthiopiens : des rois, des saints, des anonymes », Annales de Bretagne et des Pays de l'Ouest [En ligne], 114-4 | 2007, mis en ligne le 30 décembre 2009, consulté le 02 mai 2019. URL : http://journals.openedition.org/abpo/459 ; DOI : 10.4000/abpo.459 


\title{
Tombes et cimetières éthiopiens : des rois, des saints, des anonymes ${ }^{1}$
}

\author{
Marie-Laure DERAT \\ CNRS Centre d'Étude des Mondes Africains, UMR 8171
}

Christianisée dès le Iv ${ }^{e}$ siècle, l'Éthiopie se dota très tôt d'une littérature religieuse, d'abord traduite du grec, puis de composition originale. Toutefois, aucun manuscrit antérieur au xII ${ }^{\mathrm{e}}$ siècle n'a été retrouvé jusqu'à présent. À partir de cette période, il est plus facile de retracer l'histoire de ce royaume dont les souverains ont forgé l'unité autour du christianisme. L'histoire de l'Église éthiopienne est donc indissociable de l'histoire de l'État, à tel point que les lieux de conservation des textes historiques tels que les chroniques royales ou les archives juridiques, sont les bibliothèques monastiques et qu'en grande partie ces textes ont été rédigés par des ecclésiastiques. C'est au cours du Xve siècle que la production littéraire connaît un âge d'or. Pour cette raison, une grande partie de cet article portera sur cette période, avec quelques incursions jusqu'au XvIII ${ }^{\mathrm{e}}$ siècle et à notre époque.

Comme toute société chrétienne, l'Éthiopie accorde une place particulière à la mort et à son corollaire, la tombe. Celle-ci est communément perçue comme un lieu de mémoire immuable, un lieu d'éternité qui permet de perpétuer le souvenir du mort au-delà de sa propre temporalité et de celle de ses contemporains. Pourtant, cette image idéale s'observe rarement dans les faits. C'est que la tombe est l'objet d'enjeux qui sont fonction de la personnalité du mort mais dépassent parfois aussi celle-ci, si bien qu'une tombe peut disparaître au sens propre comme au sens figuré. La présence en un lieu donné d'une sépulture est fonction du souvenir qui y est attaché. Si ce souvenir s'estompe puis s'efface, la tombe ne représente plus rien. Il peut aussi arriver qu'un cimetière soit entièrement remanié pour faire de

1. Cet article est le fruit d'enquêtes menées dans le cadre d'un atelier de recherche commun sur la mort et les funérailles en Éthiopie organisé à l'Université Paris-I en 2003 et 2004, dont les résultats feront l'objet d'une publication à paraître prochainement : Bosc-Tiessé, Claire, Derat, Marie-Laure, Hirsch, Bertrand, Wion, Anaïs (dir.), Cérémonie de la mort. Études sur la mort et les funérailles en Éthiopie. 
la place ou parce que l'église à proximité de laquelle il se situe est reconstruite. Mais si une tombe peut disparaître, on peut aussi littéralement inventer un lieu de sépulture. Il advient parfois que la mémoire d'un mort soit nécessaire à la cohésion d'un groupe au point qu'il faille construire pour lui une sépulture, même s'il n'en avait pas. Les tombes et les cimetières sont donc de bons indicateurs pour juger du statut d'un mort, de l'image qu'il souhaitait laisser de lui, mais aussi de l'emploi que ses successeurs ont fait de sa mémoire.

Les sites eux-mêmes peuvent témoigner des bouleversements apportés à une sépulture, à condition qu'ils aient fait l'objet de fouilles archéologiques, ce qui est particulièrement rare pour ce qui concerne l'Éthiopie des hauts-plateaux. Mais des textes peuvent aussi remplir cette fonction dans la mesure où le mort jouit d'un statut particulièrement privilégié. S'il s'agit d'un roi, la nécropole où il sera inhumé est souvent une préoccupation de son règne et figure donc dans la chronique qui lui est consacrée. S'il s'agit d'un saint, la tombe est un élément essentiel de son culte et devient, lors des fêtes de commémoration du saint, un lieu de pèlerinage. Les héritiers du saint ont donc tout intérêt à faire connaître le lieu de sépulture du saint par le biais des légendes hagiographiques et des récits de miracles.

Cette littérature exclut le commun des mortels dont le corps n'est l'objet d'aucun enjeu. Faute de mieux, dans l'attente de recherches archéologiques, on ne peut que s'en tenir à des observations contemporaines et à des informations en creux, à partir des données recueillies au sujet des saints et des rois.

\section{L'anonymat des tombes du commun des mortels}

La seule mention historique concernant l'inhumation des morts anonymes figure dans le Livre de la lumière, une homélie du roi Zar'a Ya'eqob (1434-1468) qui présente les réformes et les idées de ce souverain en matière religieuse. Celui-ci écrit en effet :

"À propos des tombes des chrétiens, nous vous disons qu'il n'est pas permis que les chrétiens soient enterrés à l'extérieur de l'enceinte de l'église, mais seulement dans (l'enceinte de) l'église, parce que chrétien signifie église ${ }^{2}$."

Cette règle est rarement observée, pour une raison logique : l'enceinte des églises n'est pas extensible. Face au manque de place, il est donc courant d'observer des cimetières à l'extérieur des enceintes. Ceci a une conséquence directe sur la différenciation spatiale du lieu d'inhumation en fonction du statut du mort. Les plus pauvres et les plus anonymes sont rejetés en dehors de l'enceinte de l'église, tandis que les tombeaux des bienfaiteurs de l'église, des ecclésiastiques les plus fameux, sont, eux, éri-

2. Conti Rossini, C., Ricci, L., Il libro della luce del negus Zar'a Ya'eqob (Mashafa Berhan), Louvain, 1965 (CSCO 250-251, 261-262, Script. Aeth. 47-48 [I], 51-52 [II]), II, p. 24. 
gés dans l'enceinte même ${ }^{3}$. Il arrive aussi que certains se fassent enterrer dans l'église proprement dite, comme en témoignent les tombes creusées dans le roc dans l'église du Sauveur du Monde à Lalibala ${ }^{4}$. Ces tombes ont été vidées dans les années 1950 par un architecte italien qui entreprenait une restauration des lieux. Les noms et qualités des personnes qui étaient inhumées en cet endroit ont été oubliés ${ }^{5}$.

Comme partout ailleurs dans le monde chrétien, la proximité avec le sacré est recherchée, mais cette proximité est fonction du statut du mort. À Lalibala toujours, on peut observer aujourd'hui quatre lieux d'inhumation différents. Le premier est un cimetière situé à proximité des églises, constitué de nombreuses tombes anonymes parmi lesquelles émergent parfois quelques tombes plus riches, surmontées d'une croix et précisant le nom du mort (photo 1). Le second est incarné par la tombe d'un gouverneur régional, érigée au bord de l'enceinte entourant l'église de Marie (photo 2). Cette sépulture domine l'ensemble du site et est visible de très loin.

Photo 1 - Le cimetière de Lalibala pour le commun des mortels (Cliché C. Bosc-Tiessé, ACI Espaces et Territoires)

Les amas de pierres désignent l'emplacement de sépultures. Quelques tombes construites en dur émergent ça et là.

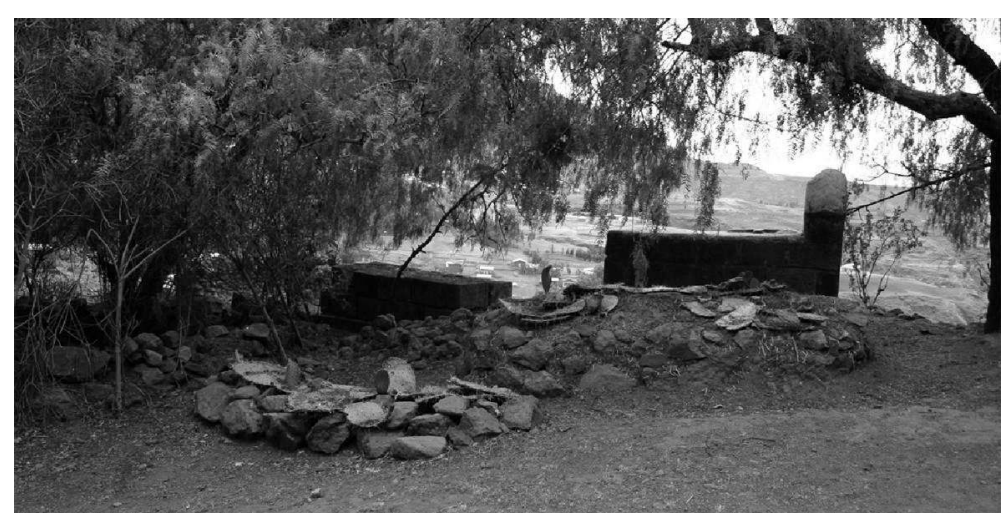

3. On peut notamment constater cette répartition dans le cimetière de l'ancienne église royale de Mashala Maryam (que j'ai visitée à plusieurs reprises entre 1997 et 2001 dans le cadre d'un programme de fouilles dirigé par Bertrand Hirsch, professeur à l'université Paris-I). Au sujet de ce programme, voir HiRSch B. et PoIssonNIER B., "Recherches historiques et archéologiques à Meshala Maryam (Manz) Éthiopie ", Annales d'Éthiopie, 16 (2000), p. 59-87.

4. Ce site est l'un des plus grands lieux de pèlerinage en Éthiopie. Fondé à la fin du $\mathrm{XII}^{\mathrm{e}}$ siècle par le roi Lalibala, il compte en tout douze églises, entièrement creusées dans la roche.

5. Ces observations ont été faites lors d'un voyage d'étude à Lalibala en avril 2005 , dans le cadre du programme ACI-Espaces et territoires (2003-2006) que j'ai co-dirigé avec Claire Bosc-Tiessé (CNRS) et qui avait pour thème : "Marges et périphéries, routes et réseaux : les processus de construction des espaces de l'État éthiopien du XIII siècle à nos jours". 
Photo 2 - Vue du site de Lalibala :

le cimetière et le tombeau du gouverneur

(Cliché C. Bosc-Tiessé, ACI Espaces et Territoires)

Au premier plan, vue du cimetière avec amas de pierres et quelques tombeaux. En arrière-plan, une partie des églises de Lalibala (couvertes de toits de tôle), dont l'entrée est la tranchée creusée dans la roche, dominée par un petit édifice, le tombeau d'un gouverneur régional.

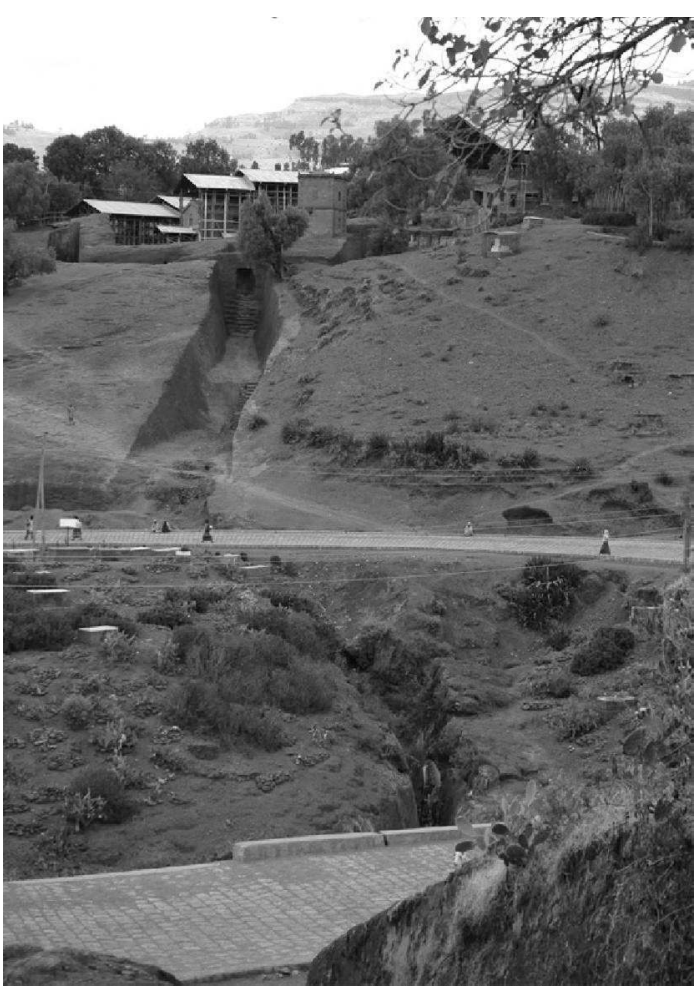

Le troisième lieu d'inhumation est celui réservé aux ermites qui séjournent encore dans les murs du sanctuaire. Ils disposent d'une cellule, trou percé dans la roche, où ils passent leurs journées et où ils sont finalement inhumés. Enfin, quelques tombes ont été aménagées à l'intérieur des églises, comme celles observées dans l'église du Sauveur du Monde et on ne peut que présumer qu'il s'agit soit de tombes d'ecclésiastiques extrêmement puissants, soit celles de bienfaiteurs laïques. Le roi-fondateur de cet ensemble, Lalibala, serait également inhumé dans l'une de ces églises ${ }^{6}$.

6. Beckingham, C. F., HuntingfoRd, G. W. B., The Prester John of the Indies. A true relation of the lands of the Prester John being the narrative of the Portuguese Embassy to Ethiopia in 1520 written by Francisco Alvares, Cambridge, 1961, p. 207. LEPAGE, C., « Un métropolite 
La qualité de la tombe elle-même reflète le statut du mort. La plupart des sépultures en Éthiopie se présentent sous la forme d'un amas de pierre. Il n'est pas rare que ces amas se superposent les uns aux autres si bien qu'une famille est en mesure de désigner approximativement l'endroit où est inhumé l'un de ses membres, sans pour autant être capable de situer précisément sa tombe. Un tel aménagement poussa la communauté arménienne présente en Éthiopie à la fin du XIXe siècle, pourtant très proche rituellement de l'Église éthiopienne, à établir son propre cimetière afin que les tombes puissent être identifiées?

Parfois aussi, le défunt a fait le vœu, avant sa mort, d'être enterré à proximité d'un lieu saint qu'il vénérait particulièrement et qui peut se situer à une grande distance de son domicile. Le monastère de Dabra Libanos, dont nous reparlerons bientôt, est ainsi un lieu très recherché pour se faire inhumer. Mais il n'est pas rare que, par manque d'argent pour transporter le corps jusqu'en ce lieu, les familles attendent plusieurs années que les ossements du défunt soient blanchis pour les emporter dans une petite caisse en bois, déposée en particulier dans une grotte naturelle située dans la falaise qui surplombe le monastère ${ }^{8}$. Le mode d'inhumation des anonymes est également très sommaire. Les cercueils sont particulièrement rares. Les corps sont donc simplement enveloppés dans un tissu et déposés dans la terre. La cavité peut être aménagée par quelques pierres, mais ce n'est pas le plus courant.

Au total, c'est donc le dénuement qui domine en ce qui concerne les sépultures des anonymes. Cet aperçu contemporain des tombes éthiopiennes est un outil indispensable pour mieux comprendre la documentation ancienne. Il est en effet plus facile d'évaluer la richesse ou la simplicité d'une sépulture en fonction de ce que l'on sait du mode d'inhumation du commun des mortels aujourd'hui. Notre documentation nous informe particulièrement sur deux types de tombes : celles des saints et celles des rois. Elles ont chacune leur spécificité, mais nous verrons qu'elles partagent parfois quelques points communs, les souverains s'étant inspirés de certains saints et les saints ayant parfois joui de funérailles dignes d'un roi.

\section{La constitution d'un cimetière monastique : les tombes des abbés de Dabra Libanos}

Le cimetière des abbés de Dabra Libanos, mis en place à partir du XVe siècle, témoigne à la fois des enjeux dans lesquels sont pris les corps des saints abbés défunts et des soins dont ils furent entourés par les souverains éthiopiens.

bâtisseur à Lalibäla (Éthiopie) entre 1205 et 1210 ", Comptes-rendus des séances de l'Académie des Inscriptions et Belles Lettres, janvier-mars 2002, p. 172.

7. Le cimetière arménien d'Addis Ababa ouvrit ses portes en 1913, cf. MERAB, E., Impressions d'Éthiopie, t. 2, La capitale et ses environs, la cour et le peuple, une page d'histoire contemporaine (1908 à 1914) : l'Abyssinie sous Ménélik II, Paris, 1922, p. 202.

8. PAnkhurst, A., "Dabra Libanos pilgrimage past and present, the mystery of the bones and the legend of saint Takla Haymanot ", Sociology Ethnology Bulletin, 1/3 (1994), p. 35-36. 
Le monastère de Dabra Libanos fut fondé à la fin du XIII ${ }^{\mathrm{e}}$ siècle par un moine, reconnu comme saint, appelé Takla Haymanot (Plante de la Foi). La communauté se développa rapidement si bien qu'à la mort du saint fondateur, en 1313, son successeur l'abbé Philippe dut déplacer celle-ci dans de nouveaux lieux afin, notamment, de séparer les nonnes des moines. De nombreux disciples de Takla Haymanot fondèrent leurs propres communautés, lesquelles se trouvaient de fait placées sous l'autorité de la maison-mère, Dabra Libanos, constituant ainsi un réseau monastique étendu. Implanté dans une région méridionale, le Choa, en cours d'intégration au royaume chrétien, le réseau de Dabra Libanos intéressait particulièrement le pouvoir royal qui voyait en lui un excellent relais. Mais jusqu'au milieu du Xve siècle, les moines refusèrent cette fonction, contestant l'ingérence excessive du souverain dans les affaires de l'Église. Cette opposition, incarnée par les abbés de Dabra Libanos, valut à quelques-uns un exil forcé, voire un emprisonnement dans les geôles royales.

À partir du règne de Zar'a Ya'eqob (1434-1468), l'attitude des abbés de Dabra Libanos s'inversa. Ce souverain sut les intéresser à une collaboration avec le pouvoir en affiliant ses propres fondations religieuses au réseau et en faisant venir à la cour deux représentants de la communauté. En échange, les abbés devinrent des soutiens indéfectibles des rois éthiopiens, se plaçant en quelque sorte comme les garants de la continuité de la royauté en cas de conflit de succession ${ }^{9}$.

L'évolution des relations de la communauté de Dabra Libanos avec le roi éthiopien se lit dans la politique funéraire menée par les moines. C'est en effet au cours du règne de Zar'a Ya'eqob, quand les conflits s'apaisent, que les moines peuvent concevoir un cimetière des abbés, dans lequel ils rapatrient les corps de ceux qui avaient été frappés d'ostracisme par les souverains. Cette politique débuta par la translation du corps de Takla Haymanot depuis son ermitage jusque dans une église construite à cet effet. Puis les moines de Dabra Libanos rapatrièrent les corps de deux abbés, Philippe (†1348) et André († 1461-1462), morts en martyrs en dehors de la communauté. Enfin, jusqu'au XVI ${ }^{\mathrm{e}}$ siècle, il semble que tous les supérieurs de la communauté furent inhumés aux côtés de leurs pères.

On ignore presque tout de la première sépulture du saint fondateur de Dabra Libanos. Sans doute fut-il inhumé dans l'ermitage qu'il s'était aménagé à la fin de ses jours, à proximité de la communauté qu'il avait fondée ${ }^{10}$. Mais il semble que cette première tombe, bien qu'en accord avec la répu-

9. DeRAT M.-L., Le domaine des rois éthiopiens (1270-1527). Espace, pouvoir et monachisme, Paris, 2003, p. 189-201.

10. Duchesne-Fournet, J., Mission en Éthiopie 1901-1903, Paris, 1909, p. 457. Bibliothèque nationale de France, Éthiopien $697, \mathrm{f}^{\circ} 68 \mathrm{r}^{\circ}$. Dans la deuxième version des actes de Takla Haymanot, rédigée à Dabra Libanos au début du XVI ${ }^{\mathrm{e}}$ siècle, l'hagiographe ne précise pas où Takla Haymanot fut enterré. Il se contente de noter que les disciples du saint " préparèrent son corps et l'enterrèrent en chantant des psaumes et des chants spirituels, ainsi qu'il doit être fait pour les prêtres" (BuDge, E. A. W., The life of Takla Haymanot in the version of Dabra Libanos and the miracles of Takla Haymanot in the version of Dabra Libanos, Londres, 1906, vol. 1, p. 233 et vol. 2, p. 94). 
tation d'ermite que Takla Haymanot s'était forgée, ne correspondait plus aux attentes de ses héritiers. La popularité grandissante de Dabra Libanos et la nécessité pour la communauté d'apparaître comme l'héritière unique du saint, face aux revendications des autres monastères fondés par ses disciples, furent sans doute à l'origine d'une première translation du corps du saint. Ce transfert eut lieu en 1370, cinquante-sept ans après la mort de Takla Haymanot. La dépouille du saint aurait alors été transportée par ses disciples depuis le lieu de sa mort jusqu'à Dabra Libanos ${ }^{11}$. Afin de prouver l'identité du corps, l'hagiographe précise que lorsque les moines ouvrirent la tombe de Takla Haymanot, un parfum agréable se dégagea, qui prouvait que le saint ne connaissait pas la putréfaction des corps ${ }^{12}$.

Dès lors la tombe du saint fut entourée d'un service particulier. Des représentants des communautés fondées par les disciples de Takla Haymanot devaient se relayer tous les mois autour de la tombe, afin d'entretenir le souvenir du saint, envoyant des prêtres pour les offices et des présents à Dabra Libanos ${ }^{13}$. Cette organisation fit naître des oppositions dans les communautés concernées qui réalisaient l'emprise de Dabra Libanos sur le culte de Takla Haymanot et constataient leur propre éviction des bénéfices de ce culte ${ }^{14}$. Celles-ci se désengagèrent donc petit à petit du service à la tombe ${ }^{15}$. Ces dissensions signalent les débats entourant l'emplacement de la sépulture de Takla Haymanot et montrent que celle-ci est parfois un enjeu entre communautés monastiques.

À la fin du xve siècle, la tombe du saint fondateur de Dabra Libanos ne faisait sans doute plus débat. Les sources manquent pour établir si les successeurs de Takla Haymanot furent inhumés aux côtés du saint, mais tout porte à croire que les abbés de la communauté étaient enterrés à Dabra Libanos. En effet, les efforts entrepris par les moines pour rapatrier les corps de deux abbés morts en dehors de la communauté témoignent de l'existence d'un cimetière des abbés de Dabra Libanos, où deux emplacements furent peut-être laissés vides dans l'attente de la translation des ossements de Philippe et André. C'est au cours de l'abbatiat de Marha Krestos, à la fin du $\mathrm{xv}^{\mathrm{e}}$ siècle, que ces translations eurent lieu. Elles interviennent au moment où la communauté n'est plus en conflit avec le pouvoir royal. Les corps des deux abbés martyrs sont donc déchargés de leur faculté de nuisance vis-à-vis de ce pouvoir.

Les ossements d'André furent les premiers à intégrer le cimetière de Dabra Libanos. Cet abbé avait été en conflit avec le roi Zar'a Ya'eqob

11. Budge, E. A. W., The life of Takla Haymanot..., op. cit., vol. 2, p. 256.

12. Ibidem, vol. 2, p. 256-264.

13. TuraEV, B., Gadla Filpos seu acta sancti Philippii, Rome, 1908 (CSCO, Aeth. 20), p. 181182/201-202.

14. DeRAT, M.-L., Le domaine des rois éthiopiens (1270-1527). Espace, pouvoir et monachisme, Paris, 2003, p. 125-134.

15. Turaev, B., Gadla Filpos..., op. cit., p. 182/202-203. Cerului, E., " Un frammento degli atti di Batergela Maryam ", Rassegna di Studi Etiopici, 3 (1943), p. 138-143. 
(1434-1468), sans doute pour son refus de reconnaître l'orthodoxie de l'observance du sabbat. Convoqué à la cour, il fut emprisonné et mourut en captivité $^{16}$. Son successeur à la tête de Dabra Libanos, Marha Krestos, fut nommé par le roi lui-même, mais d'après ses actes, il aurait refusé d'exercer sa charge tant que le corps de son prédécesseur n'aurait pas été inhumé dans sa communauté. Il aurait obtenu gain de cause et André fut enterré " avec ses pères ", retrouvant ainsi la place qui lui était due, comme l'hagiographe entend le souligner en affirmant: "Quand on le posa dans le sanctuaire, la tombe de notre père Endreyas respira trois fois comme si elle eût dit : "Gloire au Seigneur qui me fit rentrer dans mon lieu"17. "

La translation du corps de Philippe se situe dans un contexte différent. Philippe fut le troisième abbé de Dabra Libanos, entré en conflit avec deux rois du XIV ${ }^{\mathrm{e}}$ siècle. Il mourut en exil et fut inhumé dans un lieu nommé Dabra Haqalit ${ }^{18}$. Ce n'est que 140 ans après sa mort que ses ossements furent retrouvés et transférés à Dabra Libanos, avec l'autorisation du roi Eskender (1478-1494) ${ }^{19}$. Cette découverte du corps de Philippe s'apparente selon toute vraisemblance à une invention des reliques du saint. Deux témoignages sont invoqués pour prouver l'identité du saint : celui-ci se dresse dans sa tombe quand les moines commencent à creuser; on retrouve sur son cercueil un sac qui avait été employé par un de ses disciples pour tenter un premier déplacement, laissé là " afin que ce soit un signe pour le jour de la translation de notre père Philippe de crainte que ses enfants disent : "C'est notre père" ou "Ce n'est pas notre père" ${ }^{20}$ ".

Ces deux transferts, celui d'André et celui de Philippe, réalisés coup sur coup, permettent de rétablir une continuité de la lignée des abbés dans le cimetière de Dabra Libanos. Enfin le corps de Marha Krestos (†1497), en signe de l'attachement du souverain à sa personne ${ }^{21}$, après avoir été revêtu de beaux vêtements et couché dans un cercueil en bois ${ }^{22}$, chose extrêmement rare pour un simple moine, fut placé aux côtés de ses pairs dans le

16. Perruchon, J., Chroniques de Zar'a Ya'eqob et de Ba'eda Maryam, Paris, 1893, p. 100.

17. Kur, S., Actes de Marha Krestos, Louvain, 1972 (CSCO 330-331, Aeth. 62-63), p. 41-42, (traduction).

18. Turaev, B., Gadla Filpos..., op. cit., p. 220. (traduction).

19. Getatchew Haile, " The translation of the relics of abuna Fileppos of Dabra Libanos of Shoa ", Rassegna di Studi Etiopici, 34 (1990), p. 107.

20. Ibidem, p. 107-109.

21. Ces funérailles sont marquées du sceau royal et ont donc un caractère exceptionnel. Pour s'en convaincre, il suffit de rapprocher les informations concernant Giyorgis et Marha Krestos avec un épisode de la chronique du roi Zar'a Ya'eqob (1434-1468). L'auteur évoque en effet les funérailles d'un certain Takla Iyasus, fidèle du roi : " Comme preuve de sa grande amitié, le roi (Zar'a Ya'eqob) avait fait revêtir d'habits blancs et de vêtements de soie le corps de Takla Iyasus et l'avait fait mettre dans son propre caveau à Dabra Nagwadgwad " (Perruchon, J., Chroniques, op. cit., p. 172). De même, un ecclésiastique mort loin de sa communauté fut enveloppé d'un linceul, à la demande du roi Ba'eda Maryam, avant que son corps soit transporté dans sa région (ibidem, p. 134-135).

22. Kur, S., Actes de Marha Krestos, Louvain, 1972 (CSCO 330-331, Aeth. 62-63), p. 103, (traduction). 
cimetière de Dabra Libanos. La quasi-simultanéité des translations laisse à penser que les moines envisageaient la mise en place d'une nécropole.

À la fin du Xve siècle, ce programme funéraire prenait forme. Le " cimetière des abbés " était situé à proximité d'une église dédiée à Marie, construite par l'abbé Marha Krestos ${ }^{23}$. La sépulture de Takla Haymanot, le saint-fondateur, occupait une place particulière dans ce programme funéraire. Il semble en effet que le corps du saint n'était pas enterré mais que son cercueil était exposé, peut-être à l'intérieur de l'église ${ }^{24}$. En signe de piété, le roi Na'od (1494-1508) fit même transférer les ossements du saint dans un cercueil d'or ${ }^{25}$. Le cercueil était donc accessible aux fidèles, qui venaient le toucher pour obtenir une guérison ${ }^{26}$.

Si dans un premier temps, le " cimetière " des abbés de Dabra Libanos s'adressait avant tout à la vénération des moines de la communauté et aux fidèles, il prit dans un deuxième temps, à la fin du Xve siècle, une tout autre signification : les tombes des abbés, et celle de Takla Haymanot en particulier, illustraient la pérennité du monastère et devenaient un lieu de pèlerinage prisé par les souverains qui considéraient alors le saint-fondateur comme le saint patron du royaume ${ }^{27}$. Au début du XVI ${ }^{\mathrm{e}}$ siècle, les funérailles de l'abbé revêtent une grande importance. On sait en effet que le roi du moment se rendit par trois fois au monastère : la première pour assister aux funérailles de l'abbé, la seconde pour célébrer sa commémoration dans le mois qui suivit sa mort et une troisième fois, quarante jours après le décès de l'abbé, afin de nommer son remplaçant ${ }^{28}$. Dès sa mort, l'abbé était réputé comme saint puisque le roi en personne conseilla à un peintre portugais présent à la cour, qui avait perdu la vue, de se rendre sur la tombe de l'abbé décédé afin d'être guéri ${ }^{29}$. Le miracle n'eut pas lieu, mais cet épisode illustre à la fois l'attachement du souverain au monastère et la foi dans le pouvoir thaumaturgique des abbés de Dabra Libanos.

Ainsi, jusqu'en 1527, c'est-à-dire avant la guerre qui opposa le royaume chrétien aux sultanats musulmans voisins, menés par un imam surnommé Grañ - le gaucher - par les chrétiens, les abbés de Dabra Libanos furent tous inhumés dans leur monastère ${ }^{30}$. Ensuite, le cimetière périclita du fait

\footnotetext{
23. Ibidem, p. 82, (traduction).

24. Ibid., p. 81.

25. Ibid., p. 82 .

26. Dans le Livre des miracles de Takla Haymanot, on trouve le récit d'un miracle qui eut lieu au cours de l'abbatiat de Marha Krestos, au moment de la construction de l'église de Beta Maryam. L'un des ouvriers se blessa à la main et fut empêché de travailler. L'abbé lui conseilla alors d'aller toucher le cercueil en or de Takla Haymanot. L'ouvrier fut guéri et put reprendre son travail (BuDGE, E. A. W., The life of Takla Haymanot..., op. cit., vol. 2, p. 319-320).

27. Kur, S., Actes..., op. cit., p. 81-82.

28. Beckingham, C. F. et HunTingford, G. W. B., The Prester John..., op. cit., p. 261-262.

29. Ibidem, p. 262, (traduction).

30. Kur, S., Actes..., op. cit., p. 101-103; RICCI L., " Le vite di Enbaqom e di Yohannes, abbati di Dabra Libanos di Scioa ", Rassegna di Studi Etiopici, 23 (1967-1968), p. 94; BECKINGHAM, C. F. et Huntingford, G. W. B., The Prester John..., op. cit., p. 262.
} 
de l'abandon de la communauté par les moines jusqu'au XVIII ${ }^{\mathrm{e}}$ siècle. Il fallut alors réinventer la sépulture du saint-fondateur, Takla Haymanot, tandis que les tombes de ses successeurs restèrent dans l'oubli ${ }^{31}$.

\section{Enjeux autour des nécropoles royales : les sépultures des rois Zar'a Ya'eqob (1434-1468) et Ba'eda Maryam (1468-1478)}

Par certains aspects, les abbés de Dabra Libanos ont joui de sépultures dignes de rois : un cercueil en or fut donné par le roi Eskender pour les ossements de Takla Haymanot et l'abbé Marha Krestos fut inhumé dans un cercueil en bois. Or, l'emploi du cercueil semble être particulièrement rare au $\mathrm{XV}^{\mathrm{e}}$ siècle et être réservé à une élite. Une brève référence dans les actes d'un saint nous donne la mesure de la rareté de cet attribut; le saint en question était réputé pour le travail du bois, le roi Na'od (1494-1508) fit donc appel à lui pour qu'il lui fabrique un cercueil, destiné à ses propres funérailles ${ }^{32}$.

Réciproquement, les funérailles royales se sont inspirées d'éléments puisés dans les rituels destinés aux saints. Les saints étaient en effet enterrés en grand nombre dans l'église. Or, à partir du moment où les souverains fondent des églises, parmi lesquelles ils choisissent leur future sépulture, établissant parfois de véritables nécropoles familiales ou dynastiques, l'habitude de se faire enterrer dans l'église se prend. Les souverains prétendent ainsi au même statut que celui des saints, mais le sens est quelque peu différent. Si la sacralité de l'église est renforcée par la présence du corps du saint, en revanche c'est le corps du roi dont la sacralité augmente grâce à la sépulture dans l'église.

Entre la fin du XIII ${ }^{\mathrm{e}}$ siècle et la fin du XV siècle, les souverains éthiopiens ont fait construire plus d'une trentaine d'églises. Parmi celles-ci, nombreuses sont celles qui ont servi de sépulture au roi-fondateur ${ }^{33}$. C'est un cas tout à fait classique dans la royauté et sa symbolique est identique quelle que soit la région du monde dans laquelle on se situe : le souverain songeant à la perpétuation de son nom après sa mort, souhaite entourer son corps de tous les soins et des honneurs qu'exige sa fonction. Qui est mieux placé que lui pour s'occuper de tout cela? D'autant qu'il n'est pas rare qu'après la mort d'un roi, destitué, son corps soit voué aux pires traitements. Le meilleur moyen de s'en prémunir, sans toutefois réussir à contrôler ce qui se passera après la mort, est de s'occuper en personne de sa future sépulture. C'est ce que firent notamment deux souverains du Xve siècle : le roi Zar'a Ya'eqob (1434-1468) et son fils Ba'eda Maryam (1468-1478). Zar'a Ya'eqob était un souverain particulièrement autoritaire : il joua un grand rôle dans la diffusion du christianisme et sa réforme vers une orthodoxie

31. CAmpBell, I., "The church of saint Takla Haymanot at Dabra Libanos ", Sociology Ethnology Bulletin, 1/3 (1994), p. 6-7.

32. CAQuot, A., " Les actes d'Ezra de Gunda-Gunde", Annales d'Éthiopie, 4 (1961), p. 89 et 117.

33. Derat, M.-L., Le domaine..., op. cit., p. 209-219 et p. 284-300. 
proprement éthiopienne dont l'Éthiopie d'aujourd'hui est l'héritière ${ }^{34}$. Sa politique suscita de nombreuses oppositions et vraisemblablement des groupes d'opposants choisirent de soutenir ses frères ou ses fils pour tenter de le destituer et de le remplacer par leur candidat, si bien que le fils et successeur de Zar'a Ya'eqob, Ba'eda Maryam, fut accusé de trahison et emprisonné pendant un temps par son père ${ }^{35}$. Zar'a Ya'eqob, pour ne plus avoir à craindre de puissants dignitaires régionaux, choisit même de placer ses filles à la tête des gouvernements régionaux.

Ce contexte eut son influence sur la politique funéraire de Zar'a Ya'eqob. Dans la mesure où il était en opposition avec ses frères, dont certains furent ses prédécesseurs à la tête du royaume, il ne souhaitait pas être réuni à eux après sa mort. Il fonda donc sa propre nécropole royale. Mais il voulait mettre l'accent sur son lien de filiation avec son père, un grand roi du $\mathrm{XIV}^{\mathrm{e}}$ siècle, le roi David. Il prépara donc une nécropole dans laquelle il fit venir le corps de son père ${ }^{36}$ (s'appropriant ainsi son héritage) et celui de sa mère lorsqu'elle décéda ${ }^{37}$. L'un de ses fidèles nommé Takla lyasus, probablement un ecclésiastique, fut également placé dans le caveau royal ${ }^{38}$. Zar'a Ya'eqob fut ensuite inhumé avec eux. Malgré les brimades qu'il eut à subir, son fils respecta les volontés de son père. Il aurait pu choisir, cependant, de perpétuer ce lieu de sépulture royale, qui aurait alors pris un caractère dynastique lui donnant plus de prestige encore. Mais il préféra fonder sa propre nécropole, dans une autre région du royaume. Surtout, il choisit de démanteler la nécropole de son père pour fonder la sienne; il fit donc transférer dans la sépulture qu'il s'était choisie le corps de Takla Iyasus, l'ecclésiastique, se gardant bien en revanche de faire venir la dépouille de son père ${ }^{39}$. Il fit également transférer les corps de nombreux souverains, parmi lesquels figuraient le fondateur de la dynastie et quelques-uns de ses successeurs ${ }^{40}$. Il offrait ainsi à sa nécropole le statut de panthéon dynastique tout en cassant le projet de son prédécesseur.

La nécropole de Ba'eda Maryam n'eut pas non plus un grand avenir. Ses successeurs avaient bien compris l'intérêt de disposer des corps des rois morts pour rehausser leur prestige et se donner une légitimité. Nous disposons ainsi d'un exemple tout à fait parlant à la fin du xve siècle. À la mort d'un souverain, deux partis s'opposaient pour la succession, soutenant deux

34. Piovanelui, P., " Les controverses théologiques sous le roi Zar'a Ya'eqob (1434-1468) et la mise en place du monophysisme éthiopien ", dans LE BOULlUEC, A., La controverse religieuse et ses formes, Paris, 1995, p. 189-228.

35. Cerulli, E., "Un frammento... ", op. cit., p. 131-138; TAdDESSE TAMrat, "Problems of royal succession in fifteenth century Ethiopia : a presentation of the documents ", dans Atti del convegno internazionale di studi etiopici, Rome, 1974, vol. 1, p. 519-526; GETATCHEW HAILE, "The translation of the relics... ", op. cit.

36. Perruchon, J., Chroniques de Zar'a Ya'eqob..., op. cit., p. 83-86.

37. Ibidem, p. 86-87.

38. Ibid., p. 172.

39. Ibid., p. 171-172.

40. Ibid. 
candidats au trône; le parti qui l'emporta fut celui qui confisqua le corps du roi défunt, le mit à l'abri pendant la crise et lui donna des funérailles et une sépulture dignes de ce nom lorsque la succession fut enfin réglée ${ }^{41}$.

L'histoire des sépultures des souverains du xve siècle connut une dernière péripétie au cours du XVI ${ }^{\mathrm{e}}$ siècle. Des guerres opposèrent les chrétiens aux sultanats musulmans voisins (elles étaient récurrentes, mais cette foisci, les sultanats l'emportèrent et pillèrent une grande partie du royaume). Ces guerres furent suivies de migrations de populations qui vivaient jusqu'à présent plus au sud et qui occupèrent l'espace laissé libre par les chrétiens qui avaient fui les régions pillées. Dès lors, l'organisation spatiale du royaume évolua et la région centrale du pouvoir se décala vers le nordouest autour du lac Tana.

Les sépultures royales restées dans les régions occupées par des populations n'appartenant plus vraiment au royaume n'avaient donc plus d'intérêt. En même temps, les corps des souverains enterrés dans ces nécropoles étaient entourés d'un immense prestige, tout en étant détachés des enjeux liés aux querelles de succession, si bien que la nécropole de Zar'a Ya'eqob (où il restait son corps) et celle de son fils (où se trouvait notamment le corps du roi David) furent démantelées. Leurs corps furent transportés dans un monastère situé sur une île du lac Tana, un monastère particulièrement favorisé par les souverains après le Xvi siècle. Mais on ne leur donna pas une nouvelle sépulture. Ces corps furent déposés dans le trésor de l'église, où ils sont toujours exposés aujourd'hui, comme des reliques précieuses ${ }^{42}$.

Ainsi, par certains côtés, les tombes des saints et les tombes des rois partagent des points communs : les corps sont placés dans l'église, après avoir été entourés de soins particuliers (corps revêtus de fins tissus, déposés dans un cercueil) et sont l'objet d'enjeux qui entraînent bien souvent des sépultures successives, dotées d'un sens nouveau en fonction de la période. La tombe n'est pas une maison d'éternité, on adapte la sépulture à chaque époque, selon les besoins et les aspirations du moment. Les sépulcres des anonymes pourraient être dégagés de ces enjeux et par conséquent épargnés par les remaniements. Bien que l'on sache encore peu de choses à leur sujet, l'exemple des ossements de défunts que l'on laisse blanchir avant de les transférer au cimetière de Dabra Libanos suffit à montrer que ces corps entrent aussi dans des logiques de translations après la mort.

Afin de se prémunir d'un oubli bien naturel ou des remaniements de sépultures, quelques chrétiens, parmi les plus aisés, pouvaient faire don des revenus d'une terre aux ecclésiastiques desservant l'église qui jouxtait la tombe. Ce sont avant tout les souverains éthiopiens qui ont employé ce type de donation, pour la commémoration de leur souvenir, parfois aussi

41. Ibid., p. 355-356 et p. 359.

42. Ibid., p. 365; DomBrowski, F. A., Tanasee 106 : eine Chronik der Heerscher Äthiopiens, Wiesbaden, 1983 (Äthiopische Forschungen, 12), p. 157; ANNEQUIN, G., " Trésors méconnus d'une Thébaïde à l'abandon ", Les dossiers de l'archéologie, 8 (1975), p. 84. 
pour celle des membres du clergé auxquels ils souhaitaient rendre hommage $^{43}$. Mais l'efficacité de ces donations était limitée puisque les translations ont tout de même lieu, sans doute parce que le système foncier, que l'on connaît encore bien mal, évoluait et qu'une donation avait ses limites dans le temps.

\section{RÉSUMÉ}

L'histoire des tombes et cimetières éthiopiens, dans la longue durée, en est encore à ses balbutiements. Si les tombes des saints et des rois nous sont un peu mieux connus grâce à des textes témoignant à la fois des enjeux entourant les sépultures de ces personnages hors du commun et des soins apportés à leur inhumation, en revanche, les cimetières ordinaires échappent encore largement à l'enquête, en grande partie parce que le commun des mortels est inhumé dans l'anonymat et dans un grand dépouillement. C'est donc par quelques observations contemporaines que débute cet article afin de saisir ce qu'est aujourd'hui un cimetière dans l'Éthiopie chrétienne et mieux appréhender le caractère exceptionnel d'autres formes de sépultures. Puis, grâce aux textes, nous remonterons aux $\mathrm{XIV}^{\mathrm{e}}$ et $\mathrm{XV}^{\mathrm{e}}$ siècles pour suivre la création d'un cimetière monastique, et parallèlement, le développement de nécropoles royales, qui permettent de souligner comment les rites réservés aux saints et ceux réservés aux rois se sont parfois inspiré les uns des autres pour mettre en place des rituels d'exception.

\section{ABSTRACT}

The history of the Ethiopian graves and cimeteries is still very poorly studied. The tombs of the saints and kings are better known to us thanks to texts testifying the stakes about the burial of these figures out of the ordinary and the care taken concerning their inhumation. On the contrary, the ordinary cimeteries defy the enquiry because ordinary people is buried anonymously and in a great poverty. It is the reason why this article begins with some contemporary observations in order to better understand what is today a cimetery in the Christian Ethiopia and the exceptional aspect of other forms of burial. Then, with the historical texts, we will explore the $14^{\text {th }}$ and $15^{\text {th }}$ Centuries to follow the erection of a monastic cimetery and the developement in parallel of royal necropolis. These two informations allow us to underline how the rites for the saints and for the kings have inspired one another to give birth to exceptional rituals.

43. Conti Rossini C., Liber Axumae, Paris, 1910 (CSCO, Script. Aeth. 8), p. 20-21, 23-25, 27, 29-30 sqq., (traduction). 